\title{
Laparoscopic-assisted Ex Vivo Reconstruction of Renal Artery Aneurysm with Internal Iliac Artery and Auto-transplantation
}

Abdul Ahad Rana ${ }^{1}$, Brendan H. Dias ${ }^{2}$, Santosh Olakkengil ${ }^{1}$, Christine Russell ${ }^{1}$, Shantanu Bhattacharjya ${ }^{1}$

1. Surgery, Royal Adelaide Hospital, Adelaide, AUS 2. Urology, Royal Adelaide Hospital, Adelaide, AUS

Corresponding author: Abdul Ahad Rana, aahadrana85@gmail.com

\begin{abstract}
Renal artery aneurysms (RAA) represent a complex and an often incidentally found disease commonly treated with endovascular approaches. In cases where in situ approaches are unsuitable, laparoscopicassisted ex vivo repairs offer significant advantages during and post-surgery. We present a case of a female patient who presented with a long-standing right-sided flank pain. She was diagnosed with bilateral asymptomatic RAAs positioned well into the hilum, therefore making in situ repair infeasible. A laparoscopic-assisted ex vivo repair of the renal artery was performed using a graft from the internal iliac artery, which is a novel approach.
\end{abstract}

Categories: Urology, General Surgery

Keywords: renal artery aneurysm, ex vivo reconstruction, laparoscopic assisted ex vivo aneurysmectomy

\section{Introduction}

Renal artery aneurysms (RAA) are infrequently encountered [1], with a prevalence of $0.15 \%$ to $0.1 \%$ in the general population [2]. Majority of the RAAs are discovered incidentally when the patient undergoes diagnostic imaging studies for other unrelated complaints such as hypertension or back pain. While the issue of treatment of RAAs has been surrounded by significant debate, there is agreement that pregnant patients, patients with aneurysms with a diameter greater than $2 \mathrm{~cm}$, or with thrombus must definitely be treated [2]. While previously open surgical repair and endovascular approaches have been commonly used, one of the surgical treatment options that is gaining increasing popularity is the laparoscopic-assisted ex vivo aneurysmectomy [3]. This is because of its low morbidity and mortality as well as acceptable rates of consequent renal function and blood pressure control [4]. The ex vivo repair allows significant benefits especially for RAAs whose branches are not anatomically suitable for endovascular treatment or those that occur in the hilar or distal renal artery. In this report, an ex vivo reconstruction and auto-transplantation of a bilateral renal artery aneurysm has been presented, and the diagnosis, treatment, and clinical outcomes of this approach have been discussed.

Received 11/05/2018

Review began 11/14/2018 Review ended 11/17/2018 Published 11/19/2018

๑) Copyright 2018

Rana et al. This is an open access article distributed under the terms of the Creative Commons Attribution License CC-BY 3.0., which permits unrestricted use, distribution, and reproduction in any medium, provided the original author and source are credited.

\section{Case Presentation}

We present a case of a 53-year-old female who presented with a long-standing history of right flank pain. Computed tomography (CT) scan showed bilateral renal artery aneurysms measuring less than $1 \mathrm{~cm}$ on the left, and $2.1 \times 1.5 \mathrm{~cm}$ and $1.7 \mathrm{~cm}$ on the right (Figure 1 ). As is a typical case, these RAAs were asymptomatic and because of their position in the hilum, these were anatomically suitable for an in situ repair.

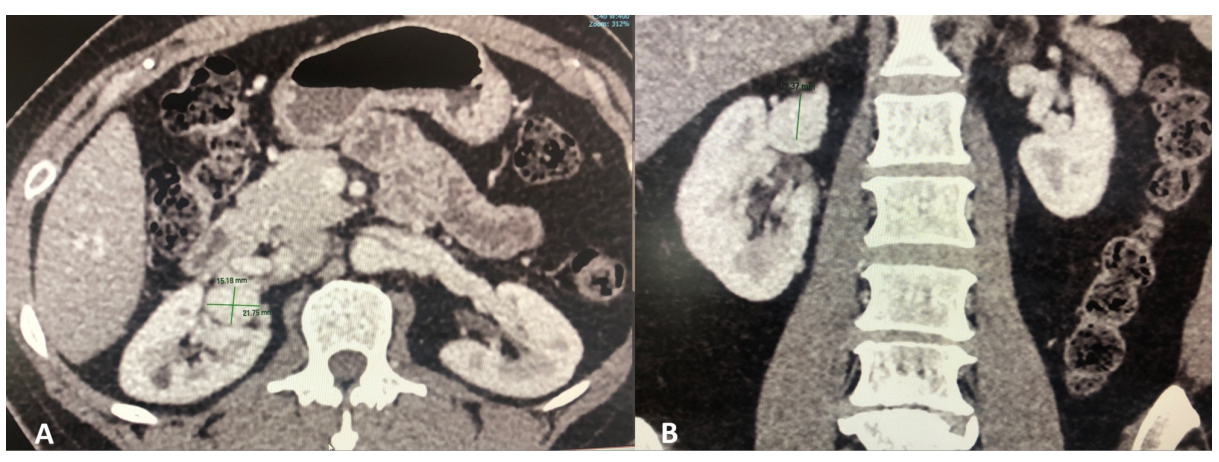

artery aneurysm measuring $21 \times 15 \times 17 \mathrm{~mm}$

CT: computed tomography 


\section{Cureus}

It was decided that an ex vivo laparoscopic reconstruction of the renal artery using a graft from the internal iliac artery was the best approach, as shown in Figures 2-4.

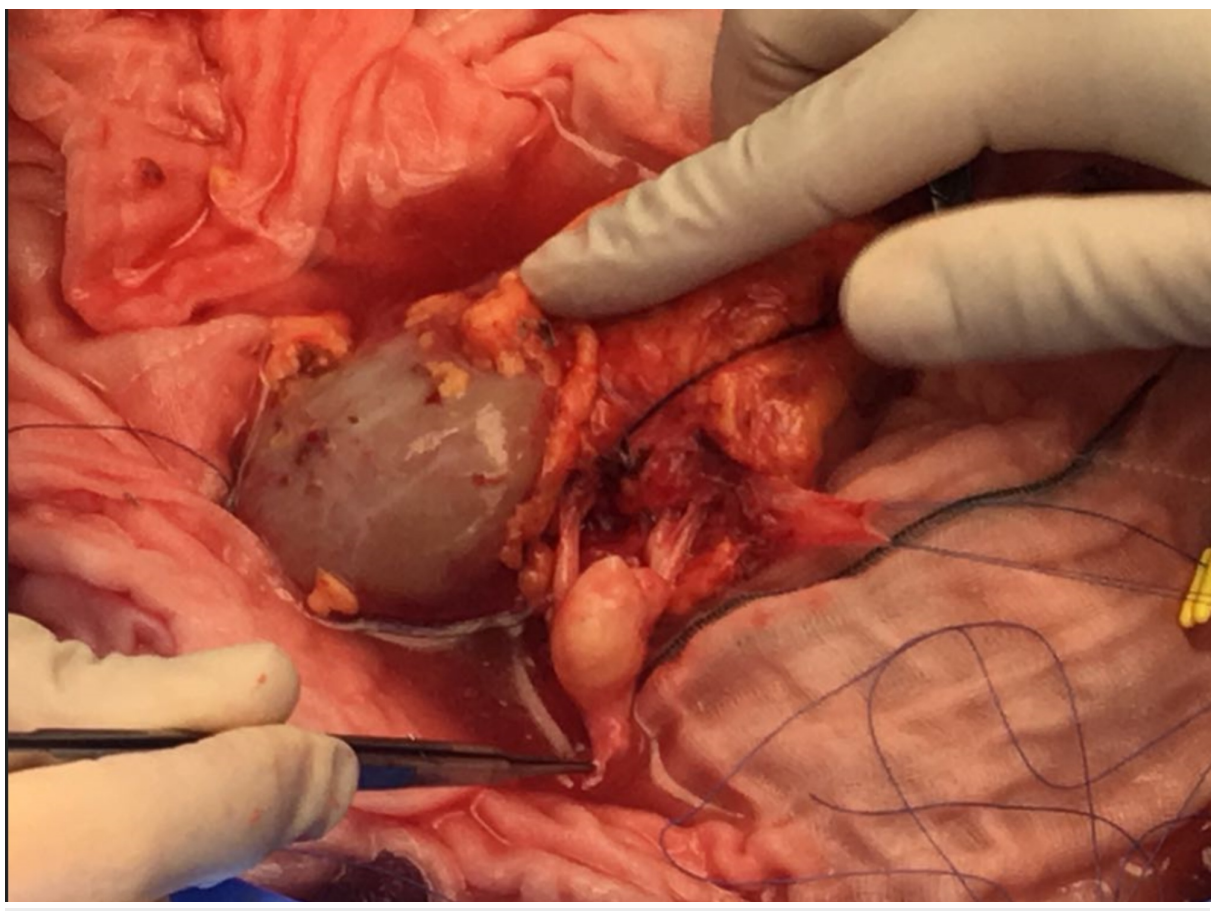

FIGURE 2: Kidney with the renal artery aneurysm

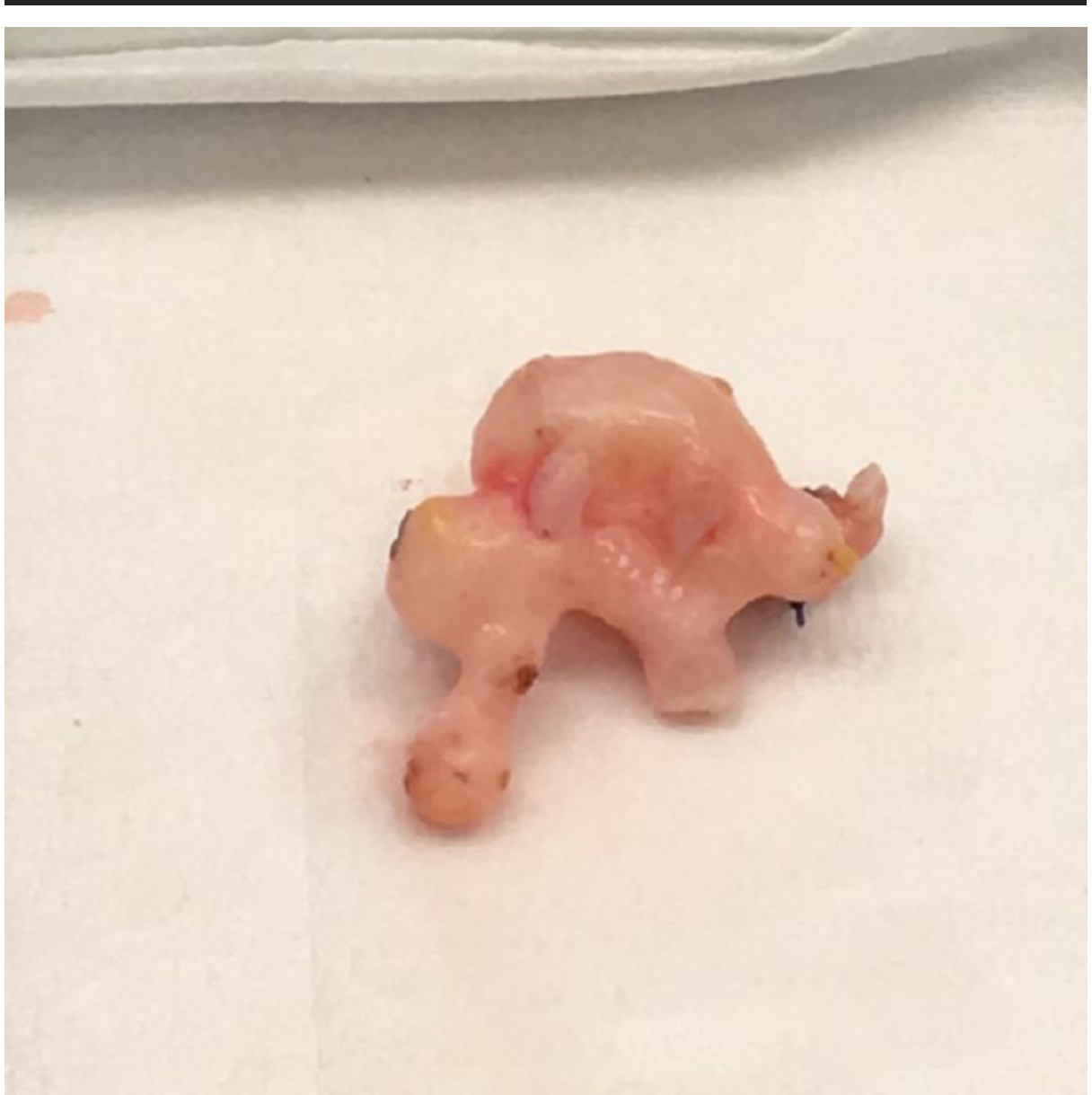




\section{Cureus}

FIGURE 3: Ex vivo renal artery aneurysm dissected

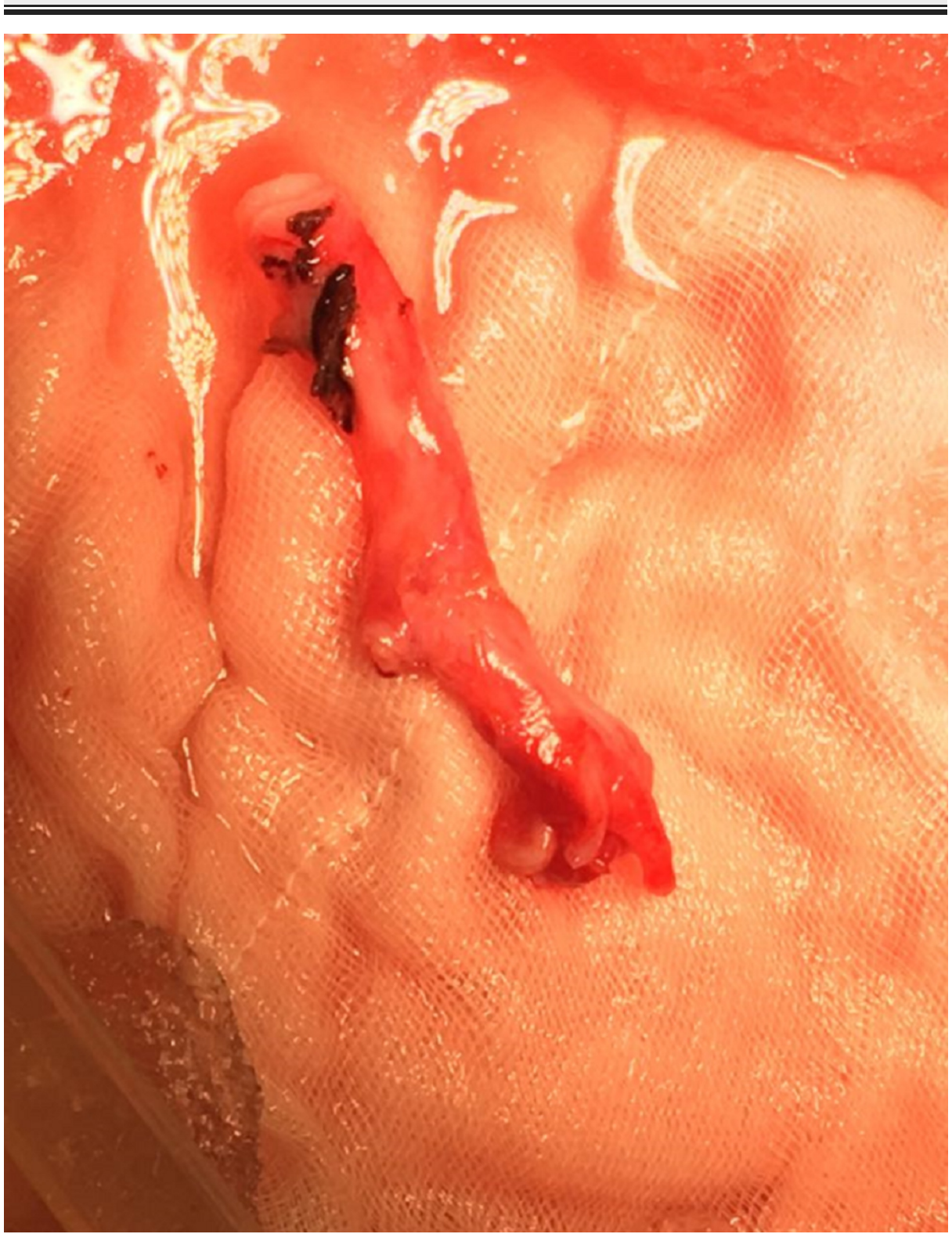

FIGURE 4: The graft of iliac artery used for renal artery reconstruction

\section{Discussion}

Endovascular surgical techniques may be used in the case of an amenable morphology and with more proximal lesions; however, in cases where the RAAs are more distal and hilar, open surgical procedures are required. In these cases, an ex vivo repair for a complex RAA is a minimally invasive procedure that allows for a smaller incision and, therefore, improved recovery times and reduced incisional morbidity [5].

Successful ex vivo renal artery reconstruction has previously been conducted and reviewed in a number of instances. For example, Ronald et al. reported reconstruction in 24 patients with a history of hypertension. Through this procedure, postoperative tubular necrosis was avoided and when observing the period between six months to six years, $95 \%$ of the patients were reported to have been cured or improved [6]. Similarly, Scherrer et al., in continuation of a series of seven successful ex vivo repairs of RAAs in donor kidneys, reported another case of a 66-year-old man, who was hypertensive despite two maintenance medications [5]. The smaller incision through the laparoscopic method allowed for an improved recovery time as well as fewer complications. Even though the patient was hospitalized again with obstipation after two weeks, a duplex ultrasound of the transplanted kidney revealed normal renal function and normal bowel movement returned in 48 hours. Consistent with these cases, our case report also revealed an improved 
recovery time and a less-invasive reconstruction of the renal artery [7].

\section{Conclusions}

Ex vivo repair and reconstruction of the renal artery provides a great potential for the treatment of most vascular lesions, especially those challenging for endovascular or open surgical options. This can allow not only limiting nephrectomy for only the severely atrophic or infarcted kidneys but also allow for a larger number of kidneys available for transplantation post-repair which would otherwise be discarded.

\section{Additional Information}

\section{Disclosures}

Human subjects: Consent was obtained by all participants in this study. Conflicts of interest: In compliance with the ICMJE uniform disclosure form, all authors declare the following: Payment/services info: All authors have declared that no financial support was received from any organization for the submitted work. Financial relationships: All authors have declared that they have no financial relationships at present or within the previous three years with any organizations that might have an interest in the submitted work. Other relationships: All authors have declared that there are no other relationships or activities that could appear to have influenced the submitted work.

\section{References}

1. Silski S, Henry ML, Rajab A, Pelletier RP: Case report: treatment of renal artery aneurysms by ex vivo renal artery aneurysm repair and transplantation. Transplant Proc. 2017, 49:2374-2377.

10.1016/j.transproceed.2017.07.010

2. Sousa P, Veiga C, Matos A, Sá Pinto P, Almeida R: Hilar renal artery aneurysm - ex-vivo reconstruction and autotransplantation. Rev Port Cir Cardiotorac Vasc. 2017, 24:185.

3. Morita K, Seki T, Iwami D, Sasaki H, Fukuzawa N, Nonomura K: Long-term outcome of single institutional experience with conservative and surgical management for renal artery aneurysm. Transplant Proc. 2012, 44:1795-9. 10.1016/j.transproceed.2012.05.037

4. Laser A, Flinn WR, Benjamin ME: Ex vivo repair of renal artery aneurysms . J Vasc Surg. 2015, 62:606-9. 10.1016/j.jvs.2015.03.070

5. Scherrer NT, Gedaly R, Venkatesh R, Winkler MA, Xenos ES: An ex vivo approach to complex renal artery aneurysm repair. J of Vasc Surg Cases. 2015, 1:165-167.

6. Stoney RJ, Silane M, Salvatierra O Jr: Ex vivo renal artery reconstruction. Arch Surg. 1978, 113:1272-8. 10.1001/archsurg.1978.01370230062007

7. Shirodkar SP, Bird V, Velazquez O, Ciancio G: Novel management of complicated renal artery aneurysm: laparoscopic nephrectomy and ex-vivo repair with heterotopic autotransplant. J Endourol. 1910, 24:35-9. 10.1089/end.2009.0338 